\title{
Supplement: Comparison of the oxygen isotope signatures in speleothem records and iHadCM3 model simulations for the last millennium
}

Janica C. Bühler ${ }^{1}$, Carla Roesch ${ }^{1}$, Moritz Kirschner ${ }^{1}$, Louise Sime ${ }^{2}$, Max D. Holloway ${ }^{3}$, and Kira Rehfeld ${ }^{1}$

${ }^{1}$ Institute of Environmental Physics, Ruprecht-Karls-Universität Heidelberg, INF 229, 69120 Heidelberg, Germany

${ }^{2}$ British Arctic Survey, High Cross, Madingley Road, Cambridge, CB3 0ET, United Kingdom

${ }^{3}$ Scottish Association for Marine Science, Scottish Marine Institute, Oban, Argyll, PA37 1QA, United Kingdom

Correspondence: Janica Bühler (jbuehler@iup.uni-heidelberg.de), Kira Rehfeld (krehfeld@iup.uni-heidelberg.de)

\section{List of Tables}

ST1 Differences between modeled and measured $\delta^{18} \mathrm{O}$ on different spatial levels. . . . . . . . . . . . . . . . 3

ST2 Improvement of median cross-correlation and SNR through the selection of the best age-model ensemble on different spatial levels.

\section{List of Figures}

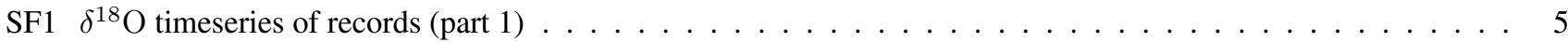

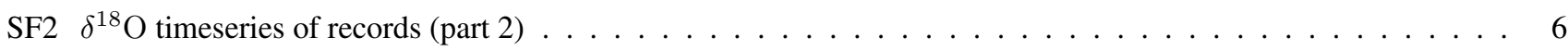

SF3 Dominant Plant Functional Types (PFT) of average june vegetation . . . . . . . . . . . . . . . 7

SF4 Systematic analysis of the relationship between variance ratio and different simulation and cave parameters $\quad \ldots \quad 8$

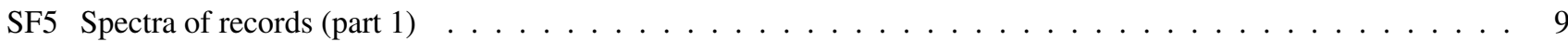

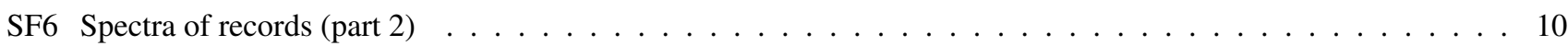

SF7 Network map and distance-correlation plot comparison between records at annual resolution simulation . . . . 11

SF8 Network maps and matrix comparison between down-sampled resolution and records; cluster Europe (c3) . . . 11

SF9 Network maps and matrix comparison between down-sampled resolution and records; cluster China and Eastern Asia (c7)

SF10 Network maps and matrix comparison between down-sampled resolution and records; cluster South America (c2)

SF11 Network maps and matrix comparison between down-sampled resolution and records; cluster North America (c1) 
SF13 Correlation map (temperature, precipitation) with strength and direction of strongest absolute seasonal correlation 14

SF14 Changing correlation distribution before and after tuning through seasonal correlation . . . . . . . . . . 15

SF15 Correlation map (temperature, precipitation, isotopic composition) showing season of strongest absolute sea-

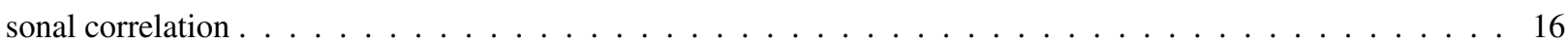


Table ST1. The difference between modeled and measured $\delta^{18} \mathrm{O}\left(\Delta \delta^{18} \mathrm{O}=\delta^{18} \mathrm{O}-\delta^{18} \mathrm{O}_{\text {dw.eq }}\right)$, corresponding to Sec. 4.1. 90\% confidence intervals were calculated via bootstrapping (1000 repetitions). Maximal/minimal outliers denote those entities with the largest positive/negative difference to the simulation.

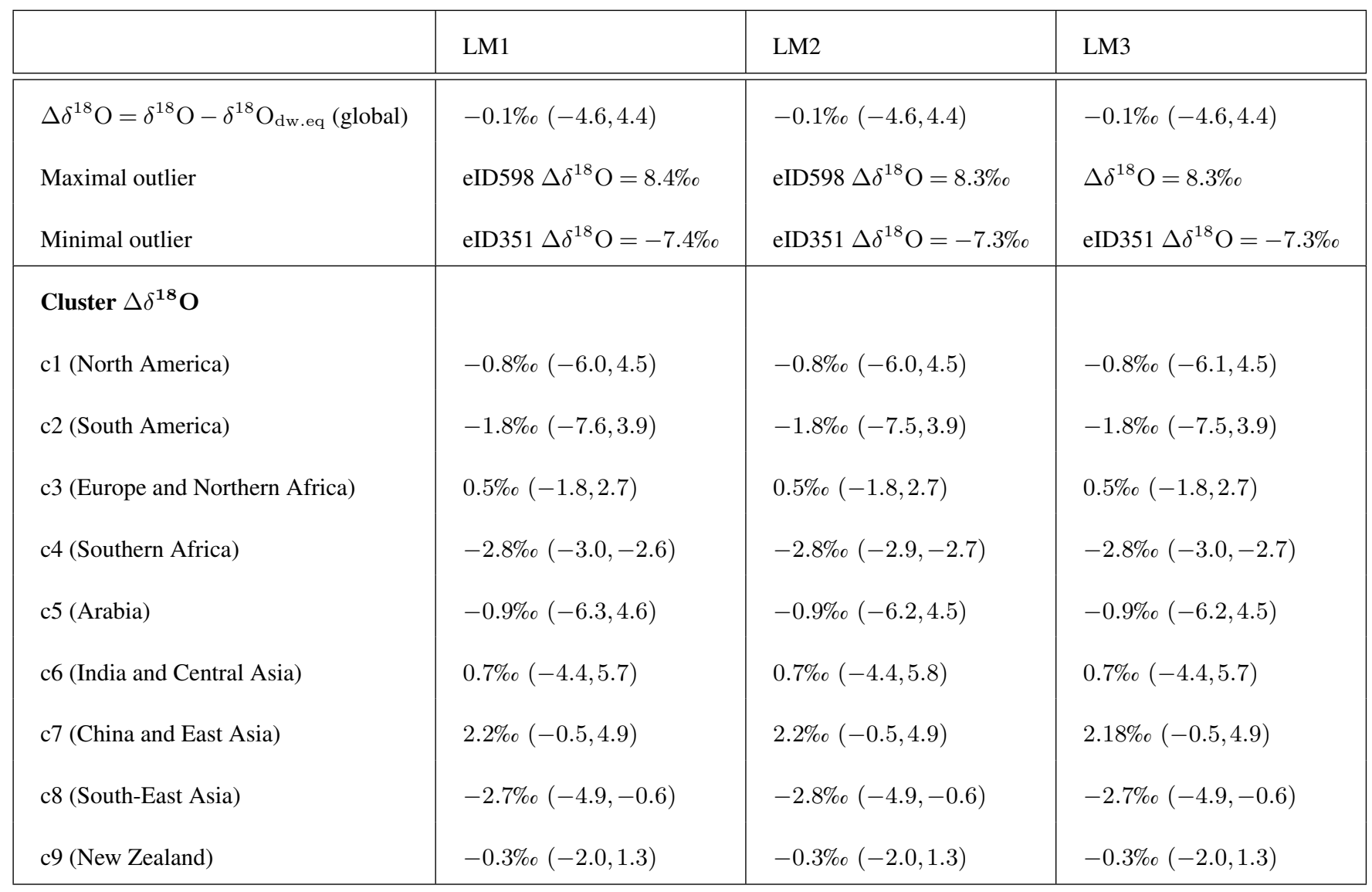


Table ST2. Improvement of median correlation $c$ and the SNR through the selection of best age-model ensemble on different spatial levels. $90 \%$ confidence intervals through bootstrapping. For c4 Africa, no age-model ensembles were available.

\begin{tabular}{|c|c|c|c|c|}
\hline Spatial aggregation level & Correlation $c$ & Age-model tuned $c_{\text {tuned }}$ & SNR & $\mathrm{SNR}_{\text {tuned }}$ \\
\hline site & $0.2(-0.0,0.3)$ & $0.5(0.5,0.5)$ & $0.3(-0.0,0.4)$ & $1.0(0.9,1.1)$ \\
\hline gridbox & $0.22(0.17,0.24)$ & $0.4(0.3,0.5)$ & $0.3(0.2,0.3)$ & $0.7(0.5,1.0)$ \\
\hline c1 (North America) & $0.1(-0.0,0.2)$ & $0.3(-0.4,0.5)$ & $0.2(0.1,0.2)$ & $0.4(0.0,0.9)$ \\
\hline c2 (South America) & $-0.1(-0.2,-0.1)$ & $0.0(-0.4,0.4)$ & $0.1(0.1,0.1)$ & $0.0(0.0,0.6)$ \\
\hline c3 (Europe and Northern Africa) & $0.1(0,0.2)$ & $-0.5(-0.6,0.5)$ & $0.2(0.1,0.2)$ & $1.1(0.7,1.5)$ \\
\hline c4 (Southern Africa) & $0.2(0.2,0.2)$ & NA & $0.2(0.2,0.2)$ & NA \\
\hline c5 (Arabia) & $0.1(-0.2,0.3)$ & $-0.5(-0.6,0.5)$ & $0.1(0.1,0.4)$ & $0.9(0.0,1.1)$ \\
\hline c6 (India and Central Asia) & $-0.1(-0.2,0.2)$ & $-0.7(-0.8,0.6)$ & $0.1(0.1,0.3)$ & $2.9(0.1,2.9)$ \\
\hline c7 (China and East Asia) & $-0.2(-0.2,-0.1)$ & $-0.3(-0.4,0.4)$ & $0.2(0.2,0.2)$ & $0.4(0.0,0.7)$ \\
\hline c8 (South-East Asia) & $-0.2(-0.5,0.3)$ & $-0.1(-0.8,0.7)$ & $0.3(0.0,1.1)$ & $0.1(0.1,3.5)$ \\
\hline c9 (New Zealand) & $0.2(0.2,0.4)$ & $0.7(0.7,1.0)$ & $0.3(0.2,0.8)$ & $2.6(1.9,76.1)$ \\
\hline global & $0.1(-0.1,0.1)$ & $0.4(0.3,0.4)$ & $0.1(0.1,0.1)$ & $0.5(0.4,0.6)$ \\
\hline
\end{tabular}




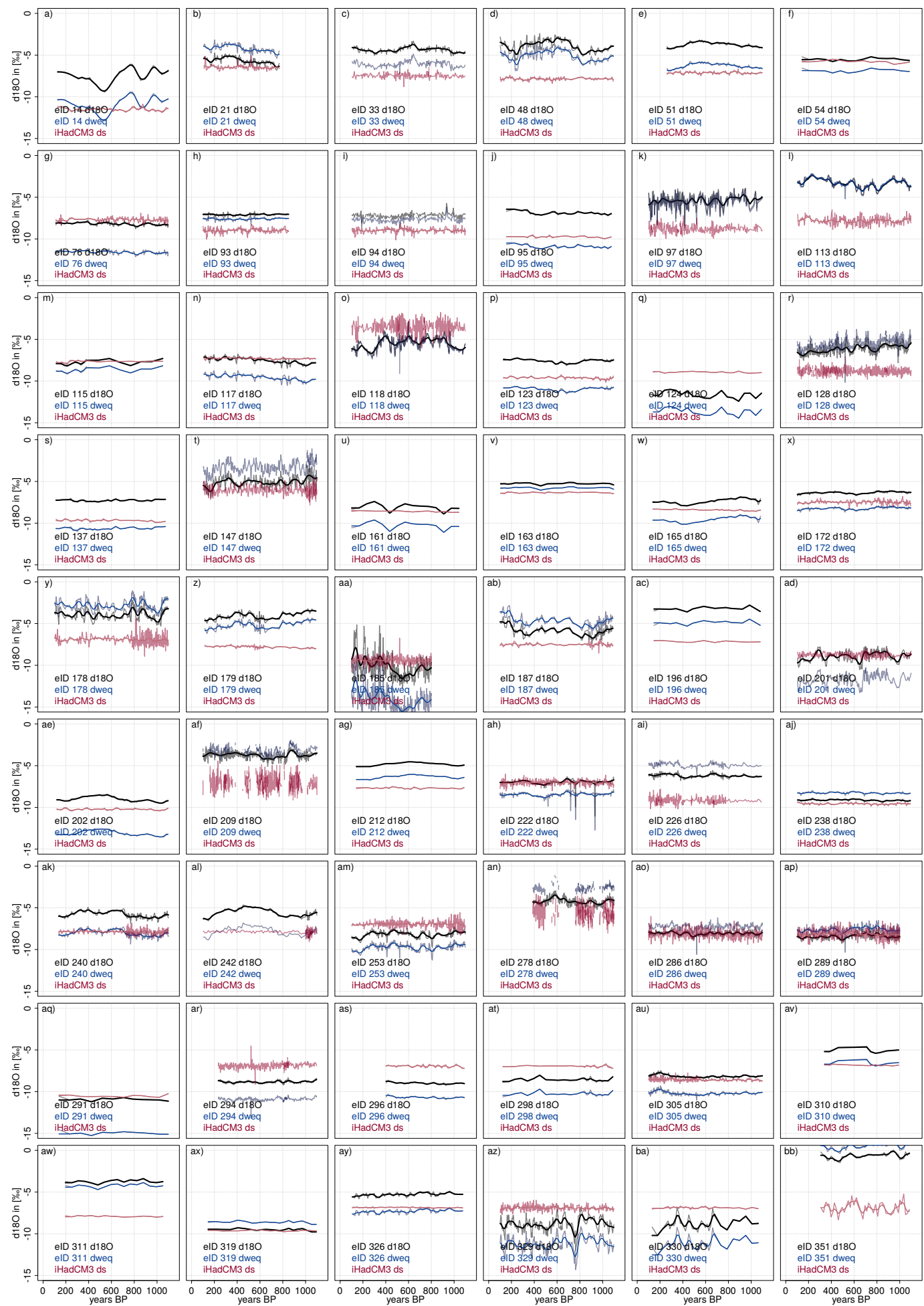

Figure SF1. Time series of all analysed records for $\delta^{18} \mathrm{O}_{\mathrm{dw} \text {.eq }}$ and for simulated $\delta^{18} \mathrm{O}_{p w}$ at the cave location (Part 1, records eID 14 to eID 351). 


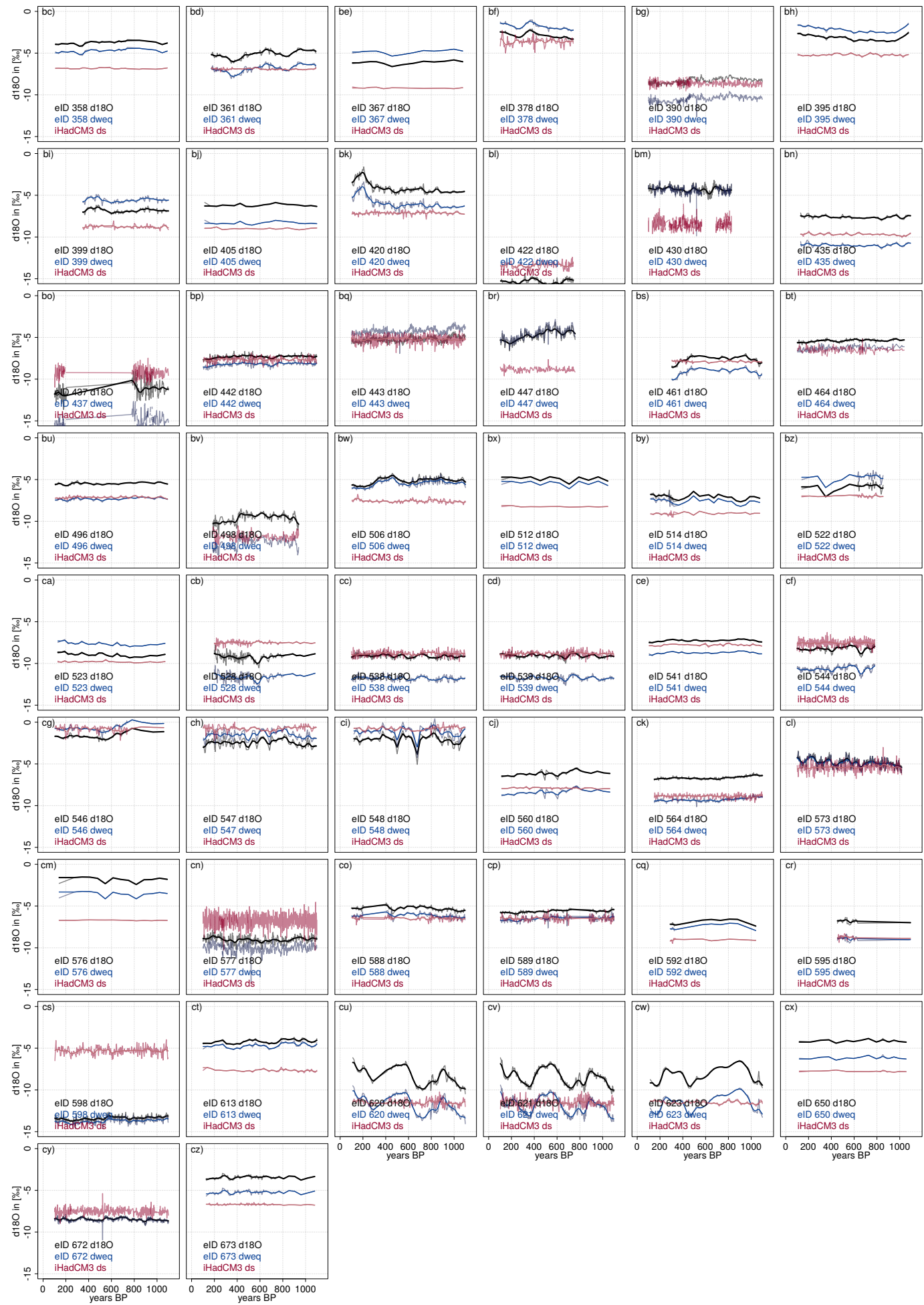

Figure SF2. Time series of all analysed records for $\delta^{18} \mathrm{O}_{\mathrm{dw} . e q}$ and for simulated $\delta^{18} \mathrm{O}_{p w}$ at the cave location (Part 2, eID 358 to eID 673). 


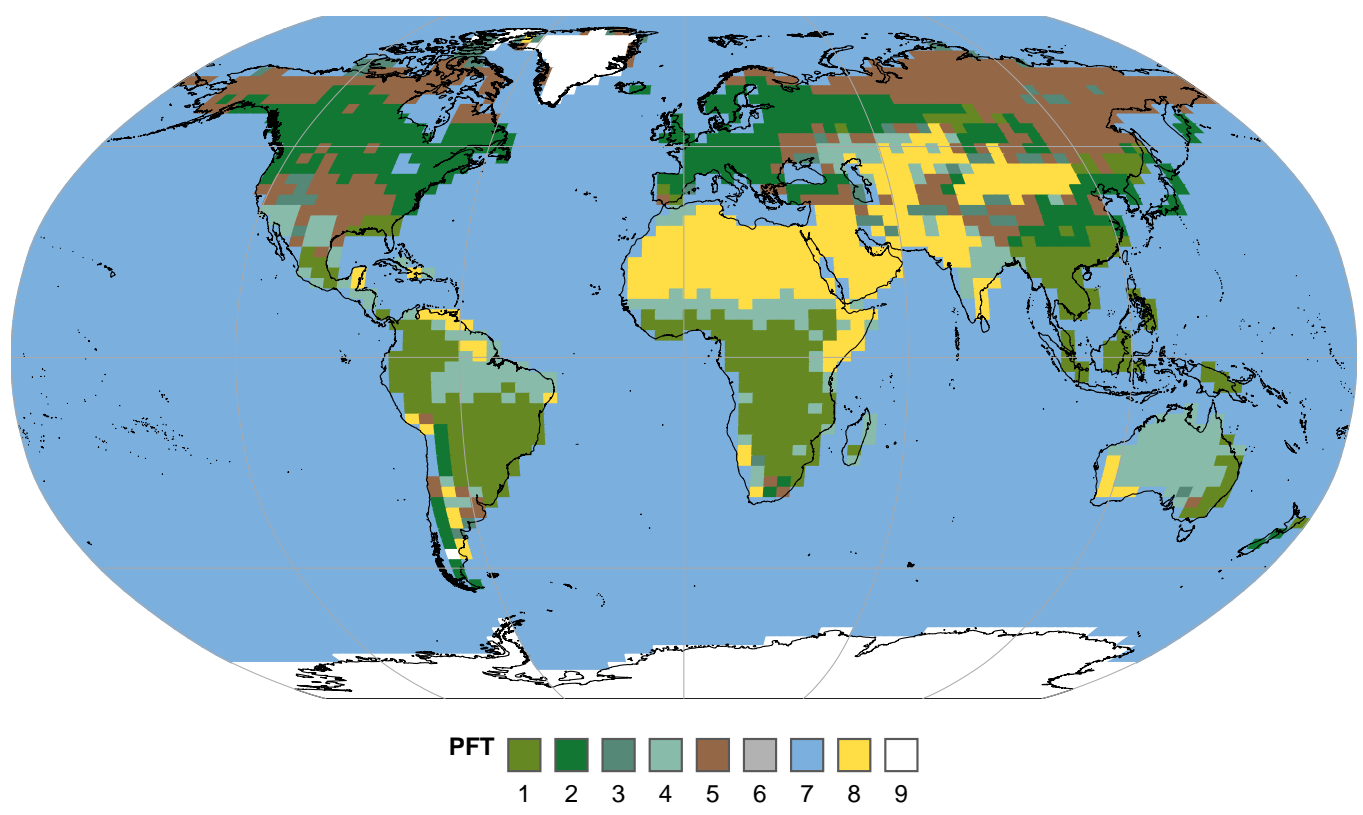

Figure SF3. Dominant Plant Functional Type (PFT) of average june vegetation: The land surface and vegetation models in iHadCM3, TRIFFID and MOSES, provide nine land surface types (broadleaf trees (1), needleleaf trees (2), C3 grass (3), C4 grass (4), shrub (5), urban (6), water (7), bare soil (8) and snow/ice (9)), where each gridbox contains a fraction of each functional type. Five of these functional types are plant types. The vegetation evolves dynamically. The plot shows the dominant PFT in each gridbox. 


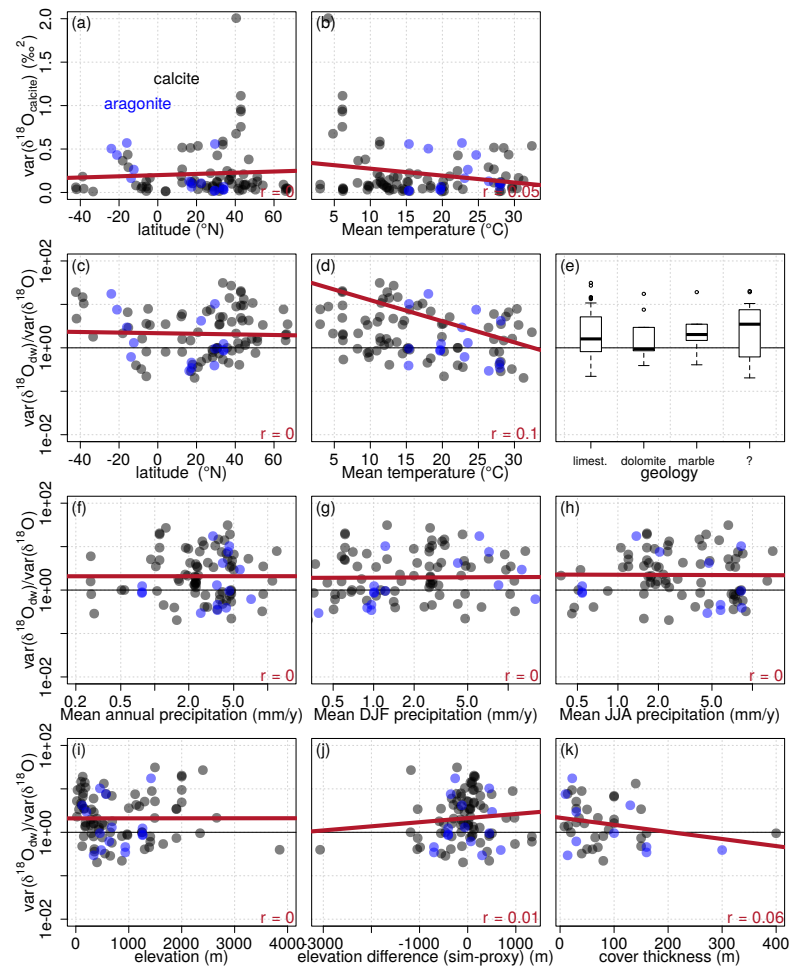

Figure SF4. No relationship between variance ratio and offset: As Fig. 4 but for the ratio of recorded over simulated variance of $\delta^{18} \mathrm{O}$ against local environmental, climatic and specimen-specific parameters. 

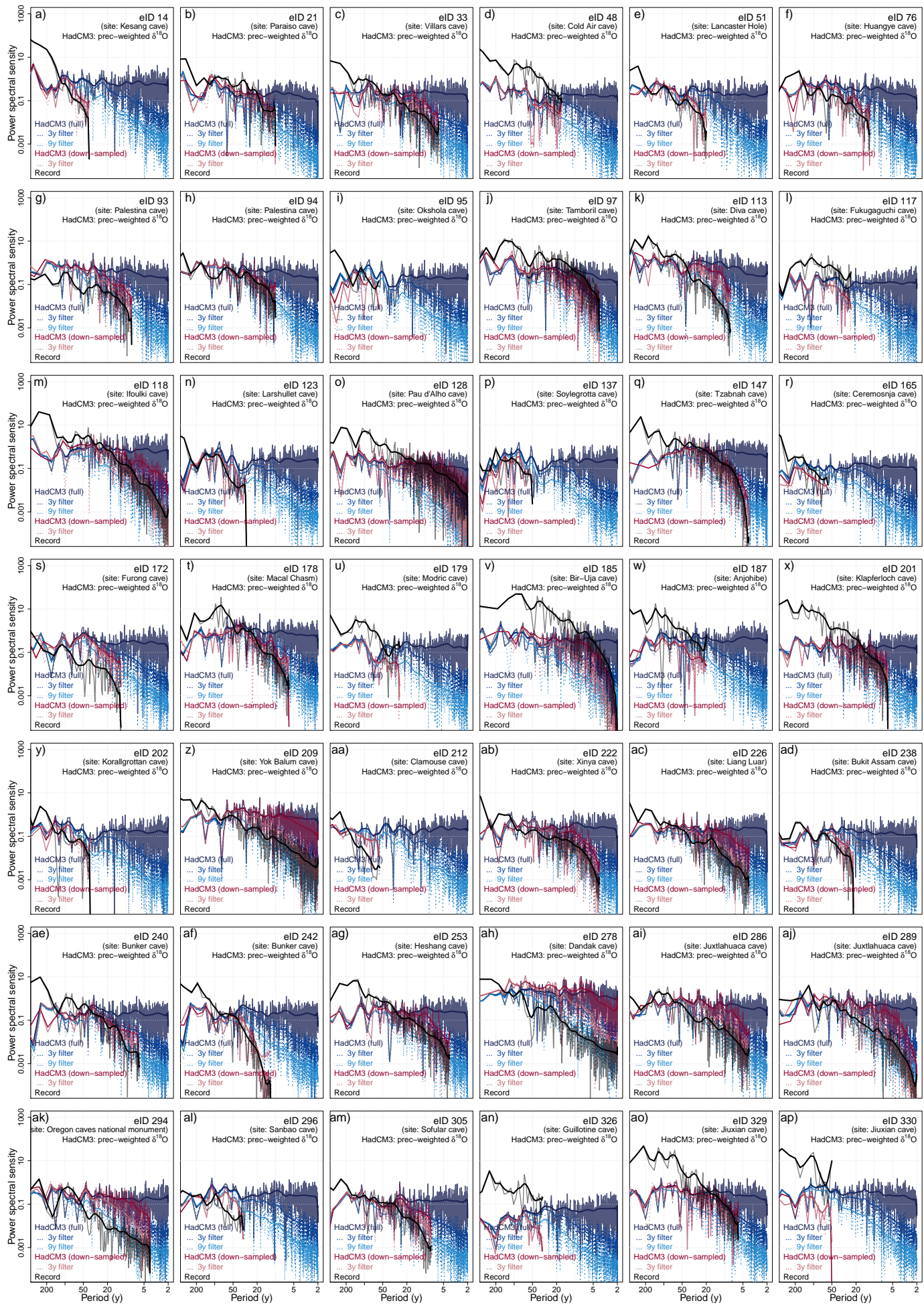

Figure SF5. Spectrum for all records used in the analysis of the yearly simulation at the cave location, down-sampled to record resolution and record with the filters corresponding to Fig. 6 (Part 1, eID14 to eID390). 

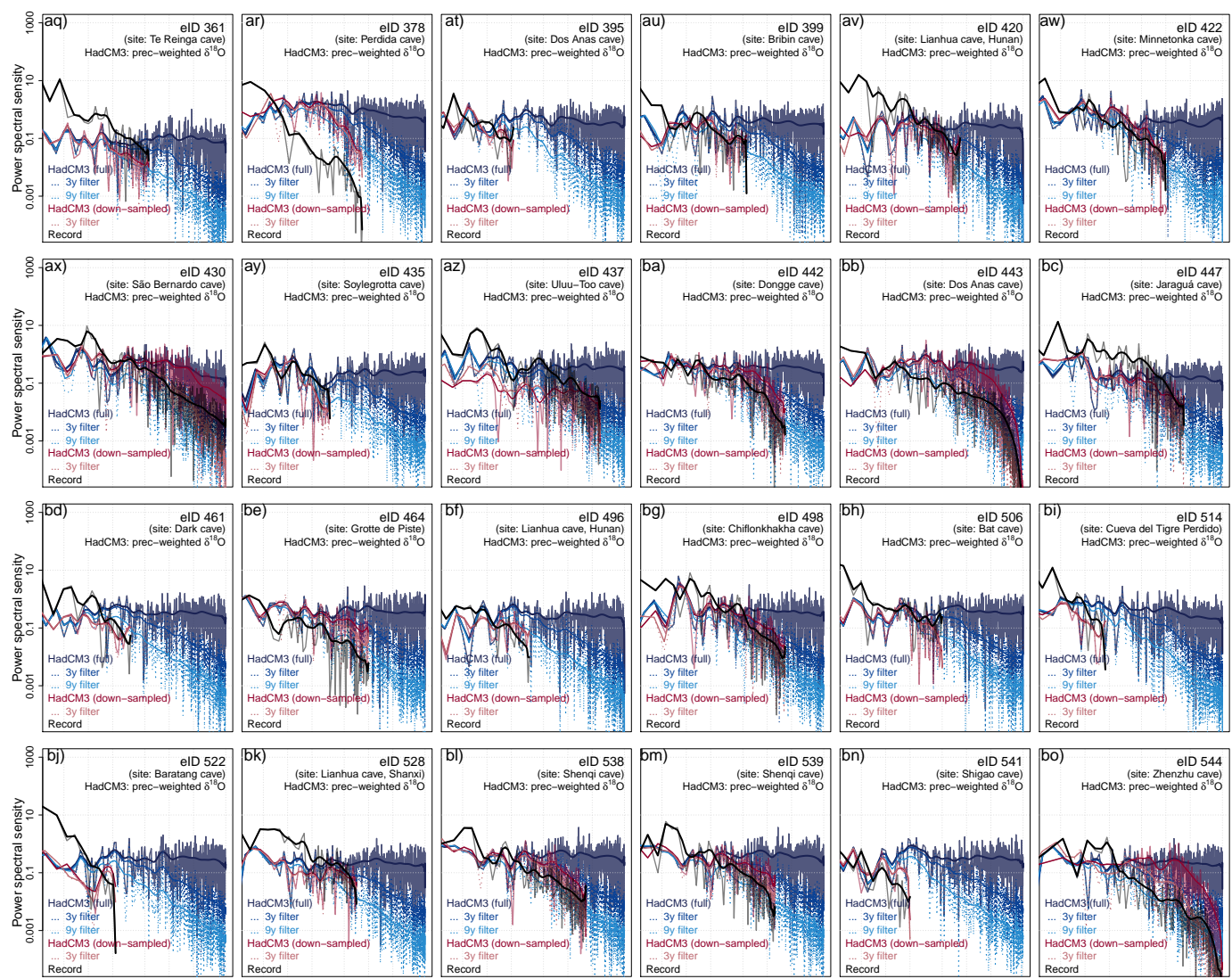

$\begin{array}{rr}\text { bo) } & \begin{array}{r}\text { elD } 544 \\ \text { (site: Zhenhnu cave) }\end{array} \\ & \text { HadCM3: prec-weighted } \delta^{\text {tio }}\end{array}$
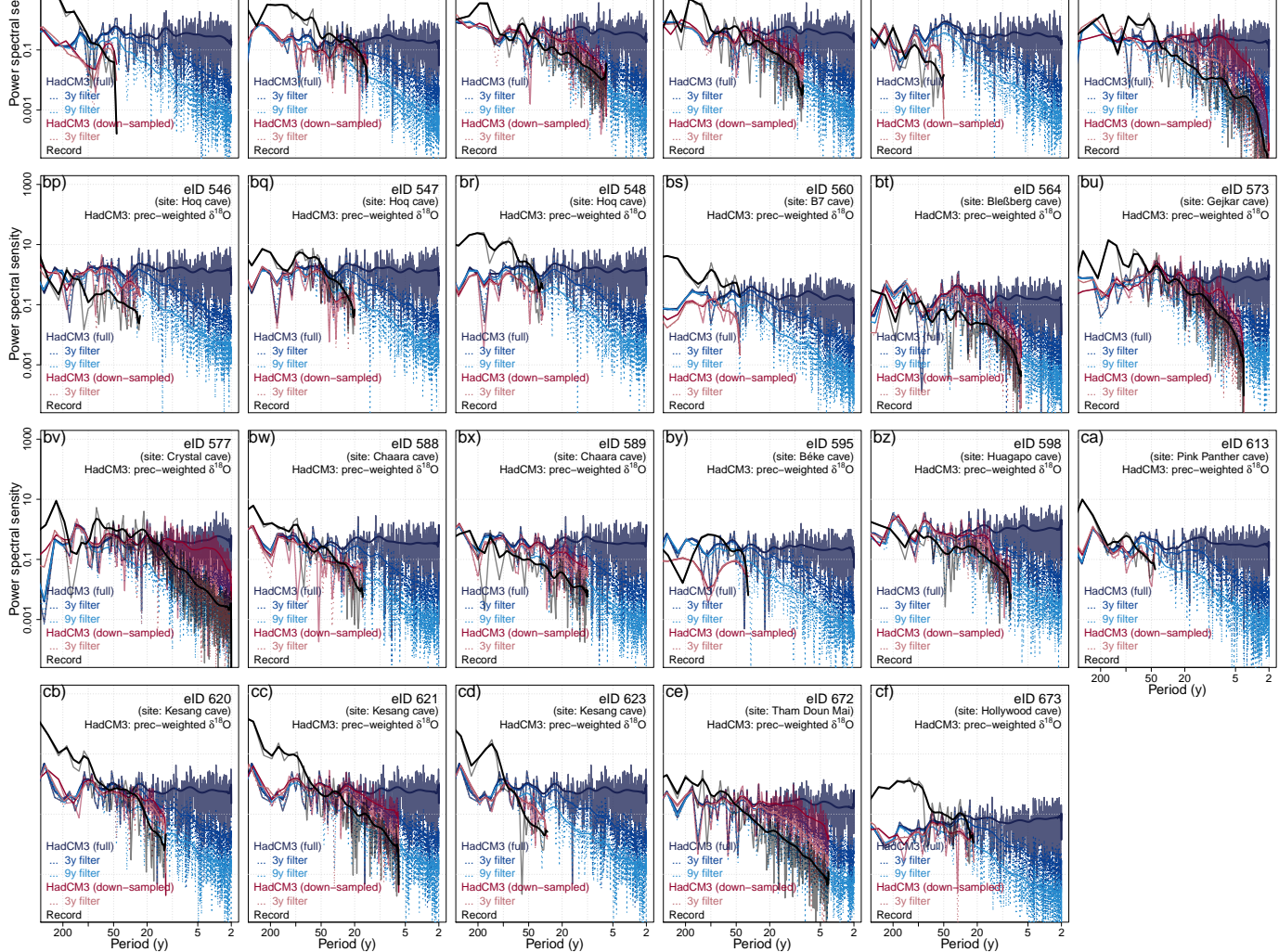

Figure SF6. Spectrum for all records used in the analysis of the yearly simulation at the cave location, down-sampled to record resolution and record with the filters corresponding to Fig. 6 (Part 2, eID 399 to eID 672). 

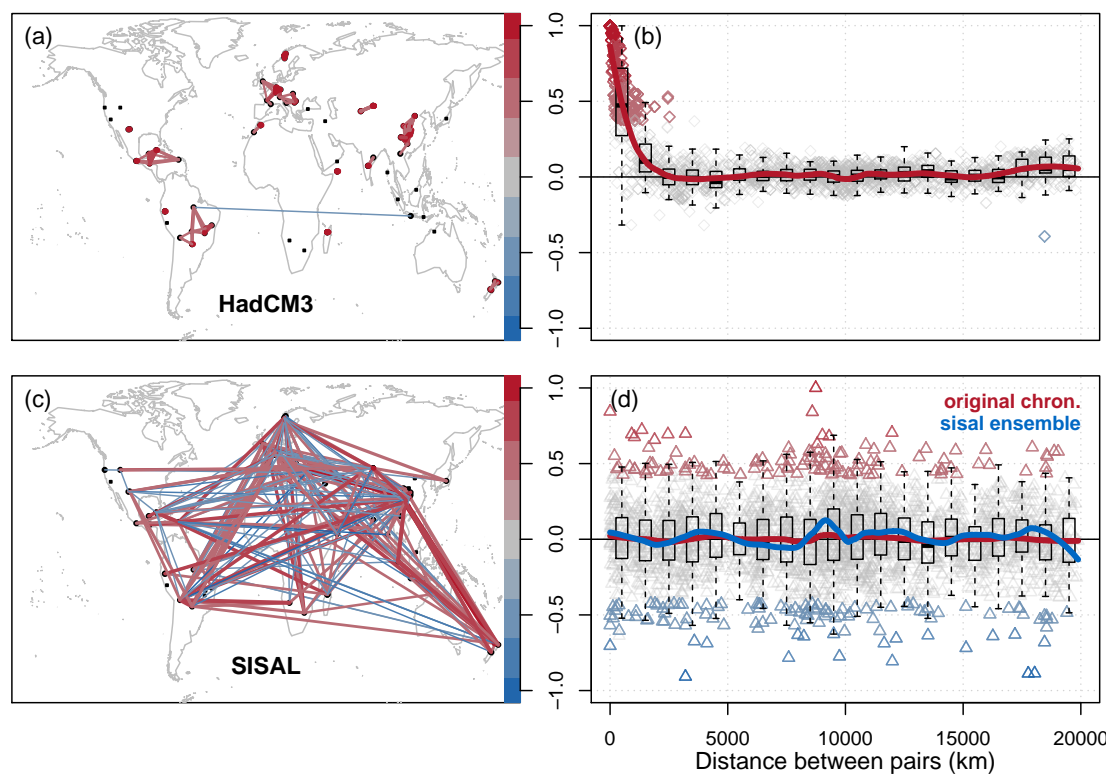

Figure SF7. Network analysis at annual resolution, corresponding to Fig. 8 in the main manuscript.
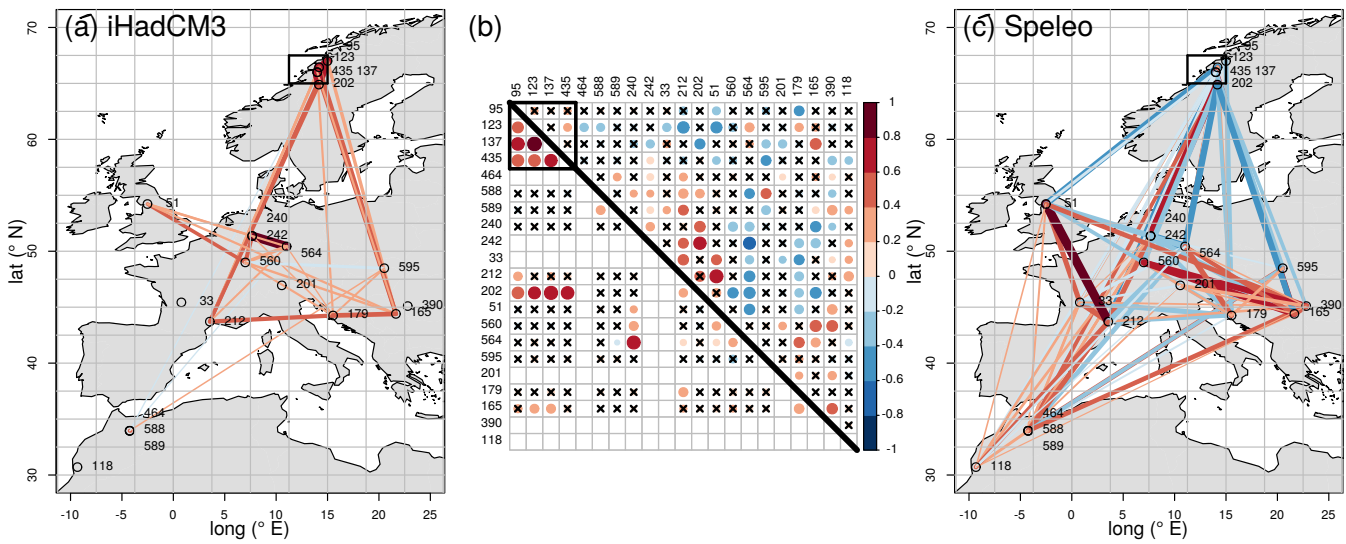

Figure SF8. Network map and matrix comparison for the Europe cluster (c3) Left map (a) shows the correlation network with the simulation data which are represented as dots in the lower triangle of the correlation matrix in the middle (b). The upper triangle in (b) and the right map (c) are based on the record to record correlation. One gridbox with 4 entities is marked in the maps and the matrix to compare directly. All links with $p<0.1$ are shown. 


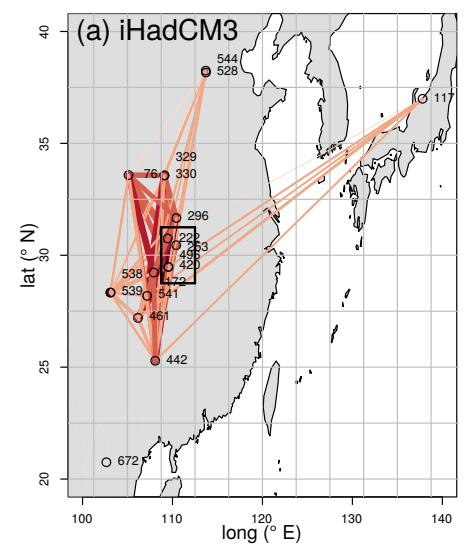

(b)

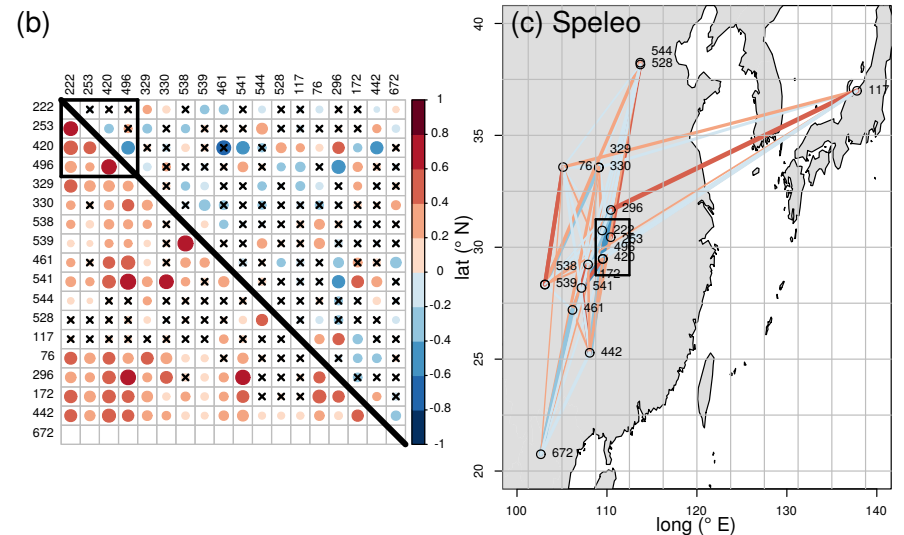

Figure SF9. Network map and matrix comparison for the China and East Asia cluster (c7) as in SF8.

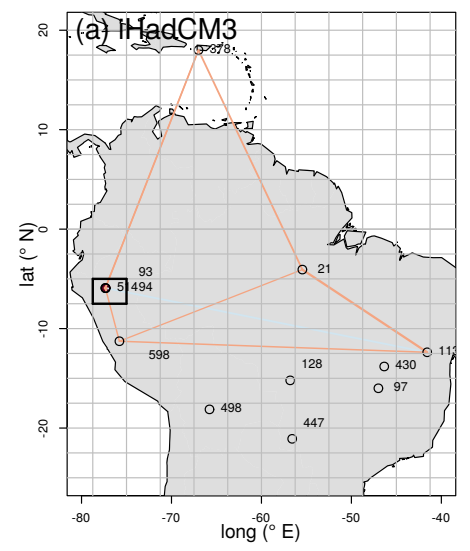

(b)

Figure SF10. Network map and matrix comparison for the South America cluster (c2) as in SF8. 

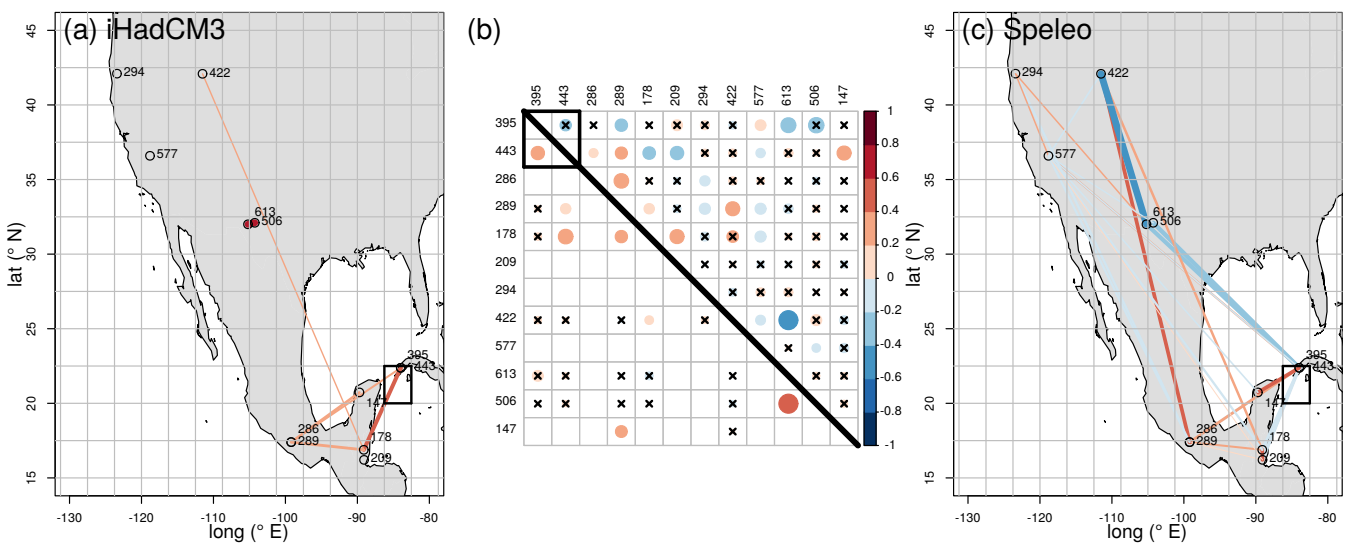

Figure SF11. Network map and matrix comparison for the North America cluster (c3) as in SF8. 

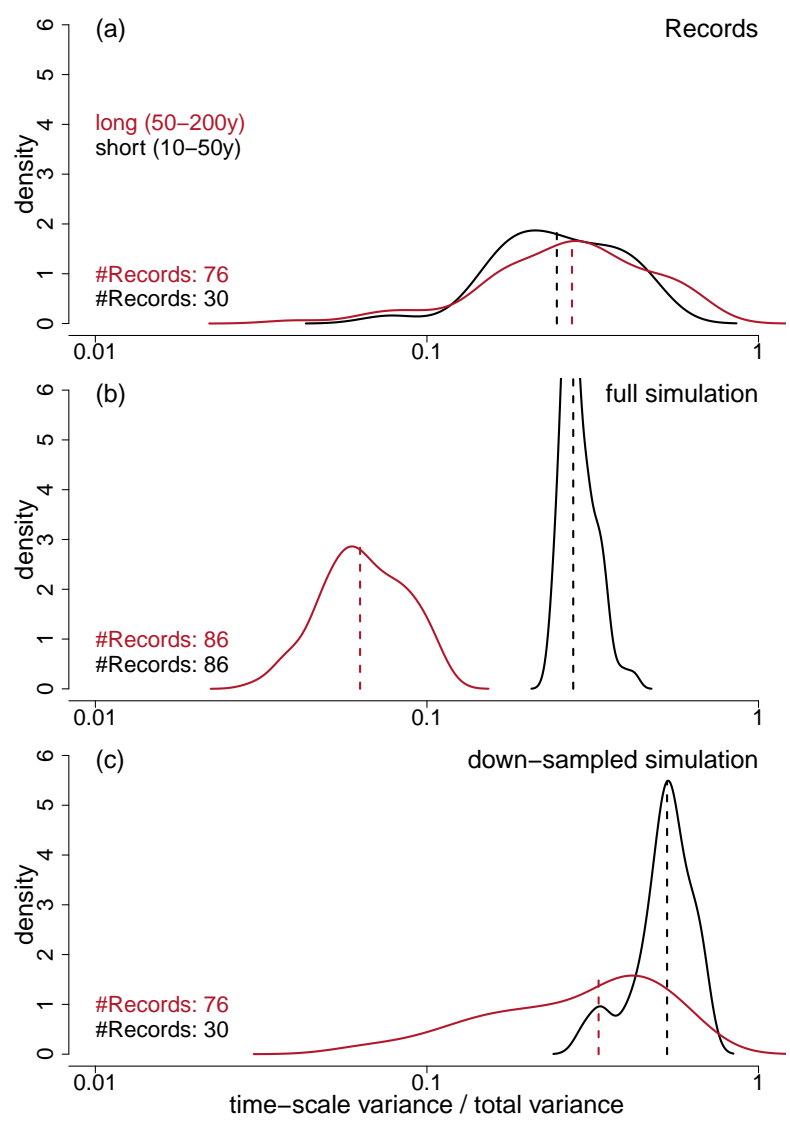

Figure SF12. Variance on different time scales: density plots of the ratio of the time period variance to the total variance for (a) the record $\delta^{18} \mathrm{O}_{\mathrm{dw} \text {.eq }}$, (b) the simulation $\delta^{18} \mathrm{O}_{p w}$ with yearly resolution and (c) the simulated $\delta^{18} \mathrm{O}_{p w}$ down-sampled to record resolution. We look at the ratio between the variance on a longer timescale of 50-200y to the total variance (red) and on shorter time scales of 10-50y (black), and show the number of records for which the variance could be extracted respectively. The dotted lines show the medians of the distribution.

(a)

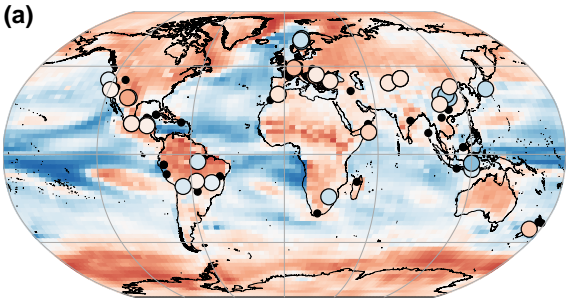

$\rho\left(T, \delta^{18} O\right)$ (b)

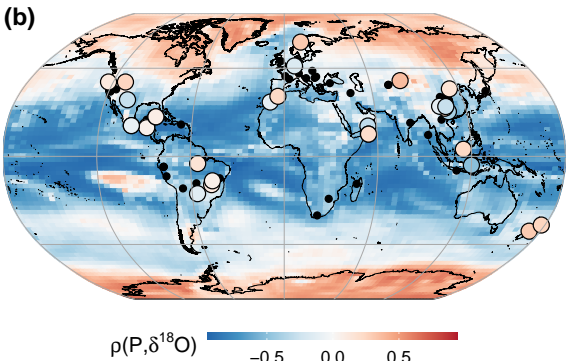

Figure SF13. Seasonal correlation: Correlation plot for temperature (a) and precipitation (b) to $\delta^{18} \mathrm{O}$ corresponding to Fig. 7, but based on correlation values obtained by tuning for the significant strongest absolute correlation of seasonal $\delta^{18} \mathrm{O}$ from $\mathrm{SF} 15$. 


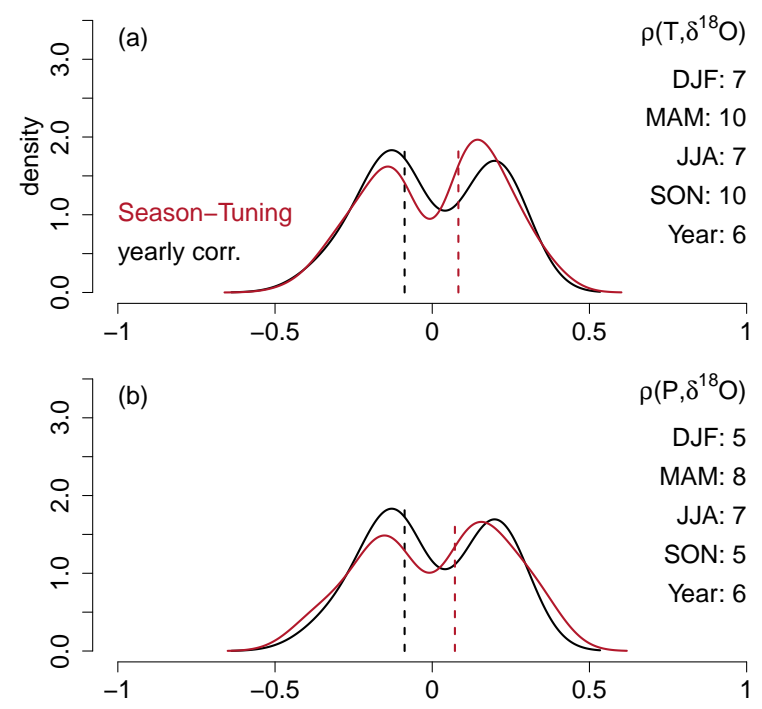

Figure SF14. Seasonal correlation optimization: Density plot showing the correlation between simulated climate parameters at cave sites, temperature (a) and precipitation (b), and $\delta^{18} \mathrm{O}_{\text {speleo. }}$. The distribution shows the correlation to the yearly signal (black) and after tuning to the seasonal signal with the absolute strongest correlation (red) as in SF15 and SF13. All simulation ensemble members are used to plot the densities. The numbers on the side indicate the number of significant correlations per season for xnapa. 
(a)

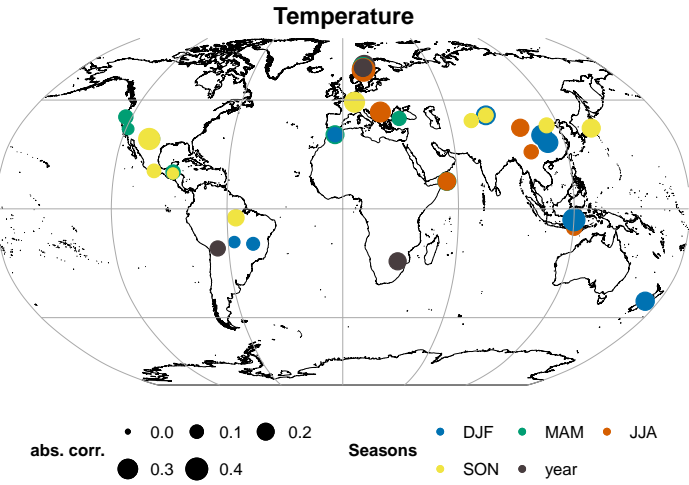

(b)

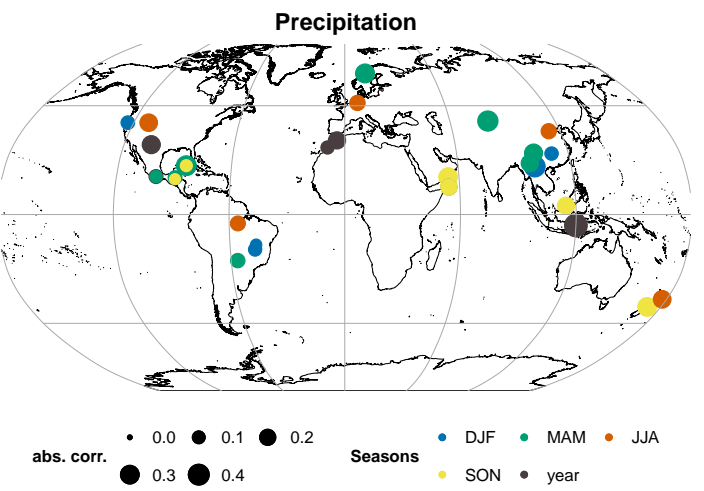

(c)

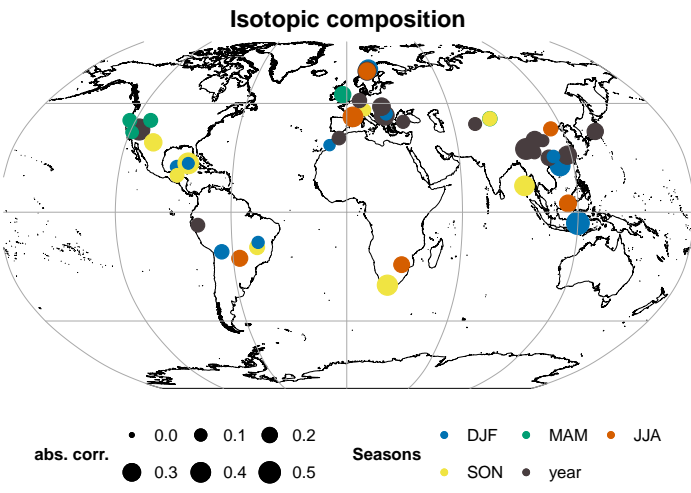

Figure SF15. Seasonal signal: Correlation between the modeled seasonal climate signal over the last millennium ((a) temperature, (b) precipitaion amount, (c) mean seasonal $\delta^{18} \mathrm{O}$ or yearly $\delta^{18} \mathrm{O}_{p w}$ ) to $\delta^{18} \mathrm{O}_{\text {speleo }}$. The dots show the abolute strength of the strongest seasonal correlation in size, and the season of strongest correlation in color ( DJF, MAM, JJA, SON). Black dots indicate where the correlation at the annual scale is strongest. 


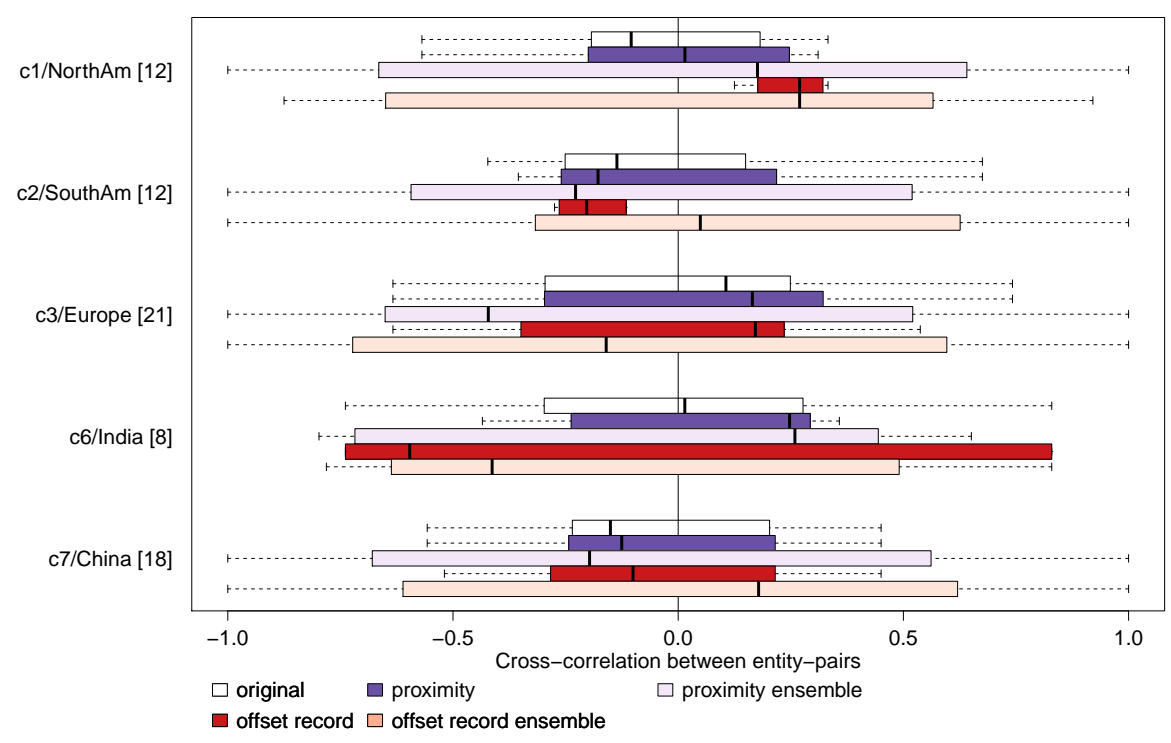

Figure SF16. Network sensitivity to age-model selection: Comparing the sensitivity of spatial correlations (original, white) to spatial proximity and mean value offsets. For the proximity test (purple bars) only the $50 \%$ spatially closest locations were considered. For testing the effect of the offsets, only the $50 \%$ of the correlation matrix with a below-average mean offset wer considered. Seeking the strognest possible absolute correlation to the entire network, positive and negative values are amplified. 